\title{
The Application of Universal Design Concept in Modern Packaging Design
}

\author{
Xiaoxiao Liu $^{1, \text { a }}$ \\ ${ }^{1}$ Zhengzhou University of Industrial Technology, Zhengzhou, Henan, 451100 \\ ${ }^{a}$ email
}

Keywords: Universal Design; Visual Communication Design; Packaging and Container Design

\begin{abstract}
Generic term was designed by Professor Mae made in 1990 to a design concept, its meaning is: to all products or commodities and the environment are designed to be, as far as possible to eliminate age differences, environmental differences and the case of ability among a by most people use a common design. Universal Design and other similar terms include: inclusive design, barrier-free design for all design. From this, universal design service object refers to all people, even the people there are physical defects, mental retardation function of the weak, the elderly and children and other groups are included, that the purpose is to give them should also provide some equal opportunity to use the facilities or products, and normal service.
\end{abstract}

\section{Introduction}

There will always be people among individuals with varying degrees of difference and distinction, even normal, will be different because of hearing or vision, or the ability to understand language and other aspects of the ability to produce more or less of some of the barriers of misunderstanding, so normal people, special groups on different levels of physiological or physical function has obstacles to mention. So, you want the full realization of universal design versatility, we need to fully take into account all the different aspects of crowd barriers and differences.

Despite or because of various aspects of a variety of reasons there is such a difference between people or another, but the main purpose is to provide general design use the same or similar manner using the same way for different users to do prevent the use of certain methods to distinguish the user, the user can not discriminate or isolate the phenomenon, in order to ensure that different users have their privacy and security.

This principle is used to emphasize that the degree of freedom can be provided various methods of using one or more users, user preferences can be based on their function required to be very freely chosen. Not only the rhythm of different users is for different types of use, but also to achieve the effect of the user feel comfortable on.

At design time, we must pay attention to whether the user is a class which will not be because of their level of education, life experience, and language abilities or limitations of different, but the feeling is hard to understand the design and operation, but to be more simple easy to understand, no complicated details, the user will be able to directly operate intuitively.

In design to convey a particularly clear and explicit user information, such as allowing: pictures, description, or other sounds can touch objects, etc., as far as possible to provide some clear information to users and the environment in which separately, to maximize the enhancement easily identify the degree of information areas, to achieve the objective of accessibility without difficulty to communicate with the user.

Pay attention to the design to maximize the impact and reduce some of the negative consequences of harmful behavior because inadvertently generated, to prevent users from adverse reactions in occasional mistakes operation, therefore, there should be reminder to guide it to the right operation or use.

Engineering principles are to design, use of movement or posture for the human body to the smallest and most effortless way to achieve operational functions. Since each user's physical condition and are different, so we need to design allows users simply to maintain a natural relaxed 
posture can achieve operational methods or procedures to reduce their fatigue.

To the extent possible to design for different users with differences in scale and spatial design objects, or as close as possible to achieve the universality of the applicable level of popularity, such as: wheelchair users, children's toys.

\section{The Application of Universal Design in Packaging}

In recent years, the use of universal design in the product and the environment has been increasingly attracted the attention of the world, resulting in universal design for the target products and services are more and more relevant. Wherein, in the product packaging and containers on the convenience of design, it has become a mainly universal design principles.

Universal Design for container packaging applications, generally have two methods: one is to design a container easy to use for everyone; while the other is the first to the elderly and people with disabilities as the main goal of the design, may ultimately It has reached all the people are easy to use design purpose. Since the second method is based on a specific design target population, you need to develop cutting-edge technology, unlike the first method, as in the face of all the people, and therefore it will put pressure on the cost to the enterprise. So now a lot of companies will choose the first method of container packaging design.

Currently on the market most of the household items are individually wrapped, when you open the lid, you need to use your fingers into the slit in the top of the box, pull the lid to open. Figure 1 below:

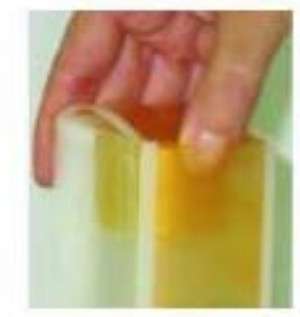

Fig. 1 Lift the lid

For this operation acts finger people with disabilities or the elderly, it is relatively more difficult. Therefore, it is necessary to design a package without opening the lid of such a design operation.

This cap with a small brim just use your fingers to push up a pulp can easily open the lid, some products will be in front of such a small brim design a U-shaped cut, so that users of pulp and small brim fit more closely, so similar design can also be used in other packaging materials, such as: plastic packaging. This design uses a common design method, without increasing the cost of the product, based on the use of convenient everyone, including people with disabilities and the elderly into account, while this design also on the back of the box, neither affect its positive appearance did not affect its function. Therefore, this universal design out of the hat with a small U-shaped incision, has gradually been adopted by many products company.

Awareness of environmental protection in the moment, has gone deep into everyone's consciousness among the Green Revolution has also begun to rise in the world, and thereby the packaging container has been designed to have a more high demands and the pursuit: the packaging of product plays a protective role, while also reducing pollution on the surrounding environment and influence. Therefore, the excessive amount appears in most of the market is accompanied by a daily refill bags, in order to reduce manufacturing and processing and packaging containers produced by carbon dioxide emissions, and resins.

Such sealed bags easy to easily open, use scissors to cut, which raises the user's objection, hoping to be easily torn by hand. Kao Corporation of Japan on this feedback, for filling thin-walled containers were analyzed to improve the analysis of such containers or pouring lip must reach four technical requirements: easy to tear mouth opened, must comply concept of environmental protection, the status quo remains the same appearance of the container, when the perfusion liquid does not overflow. 
After tests showed that in such containers as long as the general design of the four reached the technical requirements, some users objection just described will be solved, to facilitate the use of all, by the people praise.

\section{Universal Design in Visual Communication Design Package With specific reference now}

In today's era of information dissemination oriented, diversified information content and the Internet explosion has become the mainstream, into visual communication design in general has become a necessary requirement of the times. Such as traditional print, if the application of the concept of universal design, it will not only adjust the font size so simple, but will study the use of the reader when reading what kind of plate, symbol and color, etc. should be used as much as possible to avoid ambiguity. It study how to make content richer, so the accuracy of the information communicated out to meet common needs more people like visual communication design. In carrying out visual communication design, content needs to be sure to pass summarize and sort out, using distinctive visual language rich and easy to understand graphical symbols to trigger at the same time the reader's interest, but also ways to convey the desired propagation message.

Convey visual design and many times referred to as communication design, designers will be relatively difficult to understand complex information easy to understand so as to be accepted by the public. The propagation of visual symbols is no longer a one-way propagation, and spread into a multi-faceted or interact. Visual communication design under the concept of universal design is no longer just a single convey information visually, more channels from multiple senses sight, sound, touch, taste, smell and feel like passing out information. This multi-media communication mode can capture people's brains while comprehensive information to enable access to accept the multi-faceted perception and feeling, so that information more effectively achieve the purpose of propagation.

Faced with a lack of acceptance of visual communication channel with the crowd in the visual identification will be more or less there is a certain degree of disorder of visually impaired vulnerable groups, visual communication of universal design can be multi-dimensional to stimulate and assist more It means of communication to spread the message. For example: In order to avoid visually impaired people have discovered the phenomenon of eating, you can use the projection Braille so that they touch or perceived food or beverage will contain allergies become.

When public Guide system is designed, especially for some asylum or fire and other aspects of the design of the Guide will show the importance of universal design. Such visual design is through some dazzling bright colors on the three-dimensional visual symbols, special sound and multifaceted issue with the application of luminous materials to reach all kinds of people's attention and remind them to attract attention, to achieve their adaptation effects of various emergency situations.

With the increasing trend of aging, older people read newspapers, use of mobile phones and some electronic interface, are also in the progressive development of the universal visual communication design direction. Such as: When Metro Guide system design, will be on the choice of color emphasizes the strong contrast of colors, using bright colors and comfortable as a line of distinction. The aim is to give people with color blindness to bring convenience, Semanghuanzhe their eyes, because of the low ability to distinguish between colors, once the situation is not enough color to distinguish between similar or distinct encounter, their reading and recognition will be obstacles. Apply principles of universal design, designers will pay attention to the choice of colors and distinction in the design, in order to facilitate the adaptation needs of more people.

\section{Conclusion}

The development of society and civilization, it means that the members of society are supposed to enjoy this progress brings convenience and comfort. Despite the fact that the current status of development are important, in order to achieve universal design, while still just a beautiful ideals and aspirations, but it has become a goal of designers worked for. The universal design philosophy 
has always emphasized the individual can bring to every fair, so that everyone can have a participation environment, use more products, and enjoy the power of life easier. For its part, the arrival of an aging society, international integration, are showing the importance of universal design concepts and the infiltration of this design concept of the product packaging are also increasingly subject to the user's favorite companies.

\section{References}

[1] Liji Chun, Huang Qun. General design obstacle problem [J]. Packaging Engineering, 2014, (12).

[2] Guo Xinsheng, Liu Qitong. under the universal design concept identification system guides the visually impaired to explore [J]. art and technology, 2015, (10).

[3] Gong Tian. Based on application of multi-sensory concept of Universal Packaging Design [J], Beijing Institute of Printing, 2014.

[4] Zhou Jun, Deng Ying. general design concept for product development strategy inspired [J], Art and Design: Theory, 2016, (Z1).

[5] Wu Guorong, Xia Fengyu. Application of universal design principles in Preschool Children cutlery design [J]. Packaging Engineering, 2014.

[6] Zhao Chao. An aging design: inclusive stance and critical attitude [J]. decoration, 2012, (9). 